\title{
RPL24: a potential therapeutic target whose depletion or acetylation inhibits polysome assembly and cancer cell growth
}

\author{
Kathleen A. Wilson-Edell ${ }^{1}$, Amanuel Kehasse ${ }^{1}$, Gary K. Scott ${ }^{1}$, Christina Yau $^{1}$, \\ Daniel E. Rothschild ${ }^{1}$, Birgit Schilling ${ }^{1}$, Bianca S. Gabriel ${ }^{1,2}$, Mariya A. Yevtushenko \\ ${ }_{1,2}$, Ingrid M. Hanson ${ }^{1}$, Jason M. Held ${ }^{1}$, Bradford W. Gibson ${ }^{1,3}$ and Christopher C. \\ Benz ${ }^{1,4}$ \\ ${ }^{1}$ Buck Institute for Research on Aging; Novato, CA, USA \\ 2 Master of Science in Biology Program; Dominican University; San Rafael, CA, USA \\ ${ }^{3}$ Department of Pharmaceutical Chemistry, University of California, San Francisco, CA USA \\ ${ }^{4}$ Oncology-Hematology Division, Department of Medicine, University of California, San Francisco, CA USA \\ Correspondence to: Christopher C. Benz, email: cbenz@buckinstitute.org \\ Keywords: RPL24, elF6, ribosome assembly, translation, HDACs, breast cancer \\ Received: June 4, $2014 \quad$ Accepted: June 11,2014 Published: June 12, 2014
}

This is an open-access article distributed under the terms of the Creative Commons Attribution License, which permits unrestricted use, distribution, and reproduction in any medium, provided the original author and source are credited.

\section{ABSTRACT}

Partial loss of large ribosomal subunit protein 24 (RPL24) function is known to protect mice against Akt or Myc-driven cancers, in part via translational inhibition of a subset of cap(eIF4E)-dependently translated mRNAs. The role of RPL24 in human malignancies is unknown. By analyzing a public dataset of matched human breast cancers and normal mammary tissue, we found that breast cancers express significantly more RPL24 than matched normal breast samples. Depletion of RPL24 in breast cancer cells by $>\mathbf{7 0} \%$ reduced cell viability by $\mathbf{8 0} \%$ and decreased protein expression of the eIF4E-dependently translated proteins cyclin D1 (75\%), survivin $(46 \%)$ and NBS1 $(30 \%)$ without altering GAPDH or beta-tubulin levels. RPL24 knockdown also reduced 805 subunit levels relative to 405 and 605 levels. These effects on expression of eIF4E-dependent proteins and ribosome assembly were mimicked by 2-24 h treatment with the pan-HDACi, trichostatin A (TSA), which induced acetylation of $\mathbf{1 5}$ different polysome-associated proteins including RPL24. Furthermore, HDAC6-selective inhibition or HDAC6 knockdown induced ribosomal protein acetylation. Via mass spectrometry, we found that 605-associated, but not, polysome-associated, RPL24 undergoes HDACi-induced acetylation on K27. Thus, RPL24 K27 acetylation may play a role in ribosome assembly. These findings point toward a novel acetylation-dependent polysome assembly mechanism regulating tumorigenesis.

\section{INTRODUCTION}

Control of protein synthesis is commonly dysregulated in cancer, most frequently by mutational activation of the phosphoinositide 3-kinase, protein kinase $\mathrm{B} / \mathrm{Akt} / \mathrm{mammalian}$ target of rapamycin (PI3K/Akt/mTOR) pathway. The PI3K/Akt/mTOR pathway promotes cell survival and growth, by inducing the phosphorylation of the small (40S) ribosomal subunit protein S6 (RPS6) and the eukaryotic initiation factor $4 \mathrm{e}$ binding protein 1 (4eBP1). These events stimulate polysome (polyribosome) assembly and increase cap-dependent (eIF4E-dependent) translation of tumorigenic mRNAs [1-4].

Recent studies indicate that other pathways, in addition to the PI3K/Akt/mTOR pathway, can cause translational dysregulation in cancer. For example, the rRNA methyltransferase WBSCR22 is involved in the biogenesis of the $40 \mathrm{~S}$ ribosomal subunit and is overexpressed in invasive breast cancer [5]. The large ribosomal subunit protein 24 (RPL24) is another translation factor previously linked to tumorigenesis. Homozygous RPL24 deficiency is lethal in mice. In contrast, RPL24 
haploinsufficient mice are viable with specific eye, skeletal, and coat pigment defects [6]. Interestingly, these RPL24 haploinsufficient mice show greater survival from Akt-induced lymphomagenesis. This protection is associated with an overall decrease in thymocyte protein synthesis [7]. Likewise, RPL24 haploinsufficient mice are protected from Myc-driven tumorigenesis. This same study demonstrated that Myc-induced tumorigenesis arises by increased cap-dependent translation that is also prevented by RPL24 haploinsufficiency [8]. In studies of human lung adenocarcinoma cells depleted of RPL24 by RNA interference, and in RPL24 haploinsufficient mouse embryonic fibroblasts (MEFs), RPL24 reduction is associated with increased p53 expression [9], suggesting that the prevention of tumorigenesis by reduced RPL24 may also depend on a p53-dependent checkpoint mechanism.

A full understanding of the role of RPL24 in tumorigenesis requires mechanistic elucidation of how RPL24 interacts with other ribosomal proteins and translation factors. RPL24 is one of the later proteins to be incorporated into the large ribosomal subunit, where it then regulates the joining of the $60 \mathrm{~S}$ subunit to the small 40S subunit [10-12]. Crystallography of the Tetrahymena thermophilis $60 \mathrm{~S}$ ribosomal subunit and cryo-electron microscopy reconstruction of the Saccharomyces cerevisiae 60S indicate that RPL24 resides on a surface of the $60 \mathrm{~S}$ ribosomal subunit close to where the eukaryotic initiation factor 6 (eIF6) contacts the 60S [13-15]. The anti-assembly factor, eIF6, binds to the pre-60S ribosomal subunit and prevents premature association of $60 \mathrm{~S}$ with the $40 \mathrm{~S}$ subunit. Following $60 \mathrm{~S}$ maturation, eIF6 is released, allowing for the joining of the 40S sand $60 \mathrm{~S}$ subunits to form the $80 \mathrm{~S}$ ribosome and further assembly of polysomes [16-20].
Given the known role of RPL24 in murine tumorigenesis and therapeutic interest in eIF4E-driven human breast cancers [21], we asked if RPL24 expression is also altered during human breast tumorigenesis, and observed that most human breast cancers overexpress RPL24 relative to normal breast tissue. We then demonstrated that RPL24 depletion in breast cancer cells reduces their growth and viability in association with selectively impaired expression of cap-dependent proteins needed for survival and proliferation, while also inhibiting $80 \mathrm{~S}$ ribosome and polysome assembly by preventing eIF6 release from the $60 \mathrm{~S}$ subunit. We also showed that 2-24 $\mathrm{h}$ treatment with a pan-inhibitor of class I and II histone deacetylases, trichostatin-A (TSA), mimics the above effects of RPL24 depletion, inducing 60S subunit-associated acetylation of RPL24 at K27. TSA also induced acetylation of polysomal RPL24 at K93 and 14 other ribosomal proteins. Comparison of pan-, class-, and isotype-selective HDACi's suggested that HDAC6 controls total acetylation levels of ribosomal proteins, a conclusion supported by HDAC6 knockdown studies.

\section{RESULTS}

\section{RPL24 expression is transcriptionally upregulated during human breast tumorigenesis}

Since RPL24 haploinsufficiency impairs the formation of both Akt-driven and Myc-driven murine malignancies [7-9], we looked for evidence that RPL24 upregulation may contribute to human tumorigenesis as well. To that end, we compared microarrayed samples of human cancers paired with their normal organ tissue
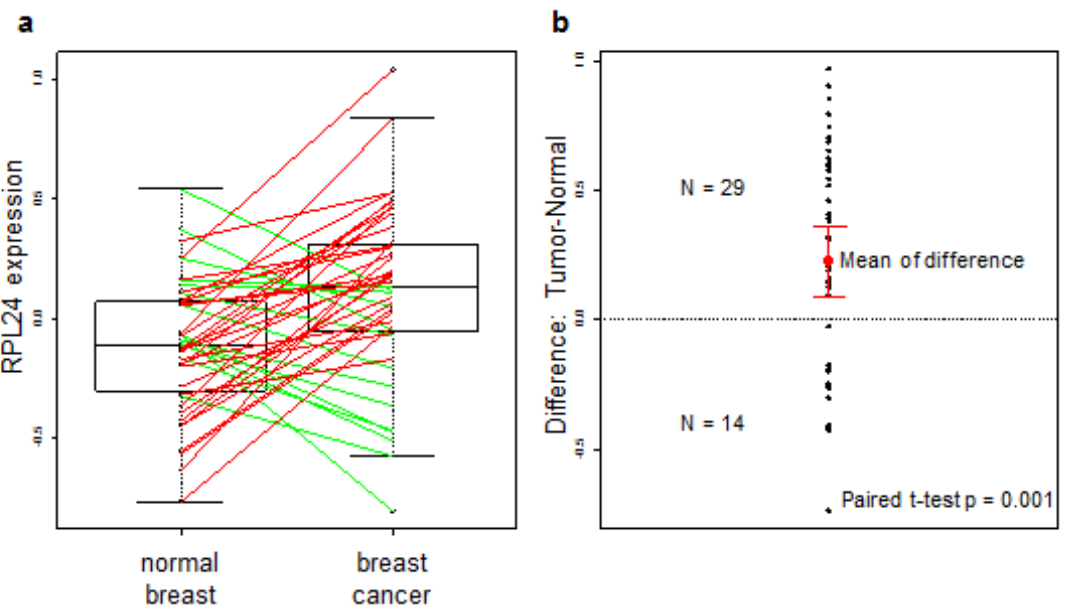

Figure 1: RPL24 expression is transcriptionally upregulated during human breast tumorigenesis. RPL24 expression levels were analyzed from the dataset presented in [22]. (a) Box plot of RPL24 expression levels in patient-matched breast carcinoma and normal breast tissues. Lines connect paired data from each patient; and line color reflects relative levels of RPL24 in each paired sample (red: tumor > normal; green: normal > tumor). (b) Differences in RPL24 expression levels between each breast carcinoma and normal breast sample pair. The mean of the differences + SD are shown in red. P-value was obtained using a paired t-test. 
samples. Using a public dataset of RNA profiles reported from 43 pairs of breast cancer and normal breast samples [32], we determined that approximately two-thirds of the breast cancers showed increased RPL24 transcript levels relative to their matched normal breast sample (Figure 1a). The entire group of tumor samples exhibited a significant $20 \%$ mean overall increase in RPL24 expression levels $(\mathrm{p}=0.001)$, indicating that transcriptional upregulation of RPL24 commonly occurs in human breast tumorigenesis (Figure 1b).

\section{RPL24 knockdown reduces breast cancer cell viability while inhibiting cap (eIF4eE)-dependent expression of proliferation, survival and genome stability proteins}

Studies of RPL24 haploinsufficient mice protected from Myc-driven tumorigenesis revealed that dysregulated cap-dependent protein synthesis not only induces tumor formation but also results in cell cycle dysregulation and genomic instability [8]. Since the translation-dependent checkpoint mechanism remains undefined, we evaluated the impact of RPL24 depletion in a model human breast cancer cell line, SKBR3, sensitive to eIF4E-regulated and cap-dependent translation inhibition [33]. Two different RPL24-directed shRNA-expressing lentiviruses were used to decrease RPL24 protein expression by approximately $70 \%$ (Figure $2 \mathrm{a})$. This resulted in a 5 -fold $(80 \%)$ reduction in SKBR3 cell viability measured after 4 days in culture (Figure 2b). Associated with RPL24 depletion and growth inhibition was a marked reduction in the expression of three different eIF4E-regulated and cap-dependent transcripts necessary for cell proliferation $(75 \%$ reduction in cyclin D1), survival (46\% reduction in survivin), and DNA repair and integrity (30\% reduction in NBS1). Protein levels of two housekeeping genes not regulated by eIF4E [21, 33-35], GAPDH and $\beta$-tubulin, were not affected by RPL24 depletion (Figure $2 \mathrm{a}$ ).

\section{RPL24 knockdown reduces $80 \mathrm{~S}$ and polysome assembly while increasing $60 \mathrm{~S}$ retention of eIF6}

Since RPL24 depletion decreased the levels of three cap-dependently translated proteins, we next evaluated the impact of RPL24 knockdown on overall ribosome and polysome formation in these cells. We used polysome profiling, which utilizes continuous sucrose gradient fractionation to separate free $40 \mathrm{~S}$ and $60 \mathrm{~S}$ ribosomal subunits, $80 \mathrm{~S}$ ribosomes, and polysomes (two or more ribosomes on one mRNA). The ratio of both $80 \mathrm{~S}$ ribosomes and polysome peaks relative to free $40 \mathrm{~S}$ and $60 \mathrm{~S}$ ribosomal subunits was significantly reduced

b

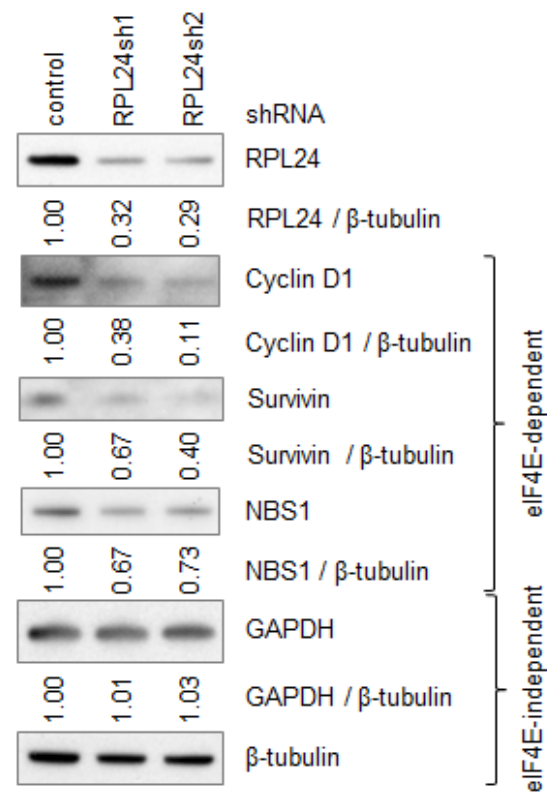

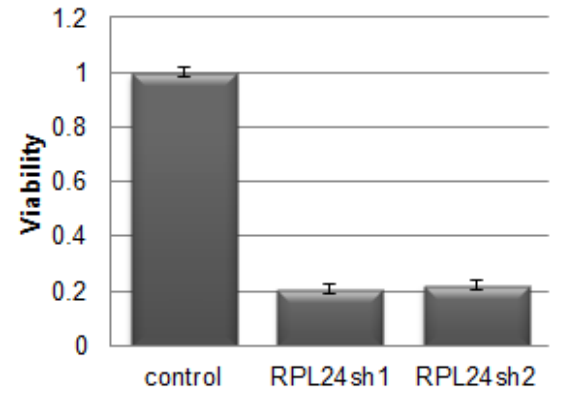

Figure 2: RPL24 knockdown reduces breast cancer cell viability while inhibiting cap (eIF4E)-dependent expression of proliferation, survival and genome stability proteins. SKBR3 cells were infected with lentiviruses expressing a GFP control or RPL24-targeting shRNA. After one week of puromycin selection, cells were plated in 96-well plates for viability assays and lysates were taken in parallel for western blots. (a) Western blots were performed on lysates from an equal number of cells using antibodies toward the indicated proteins. Ratios of protein expression normalized to beta-tubulin levels were obtained using ImageJ software. (b) Viability assay readings were taken three hours after plating (day 0) and four days after plating (day 4). The day 4 results were normalized for plating efficiency using the day 0 values. Error bars represent three replicate samples. 
in SKBR3 cells following efficient RPL24 knockdown (Figure 3a,b). This observed increase in $40 \mathrm{~S}$ and $60 \mathrm{~S}$ subunits relative to $80 \mathrm{~S}$ ribosomes implies a defect in 40S-60S joining induced by the RPL24 knockdown. Since eIF6 bound to the pre-60S subunit prevents joining of the 40S and 60S subunits [16-20] and occurs adjacent to RPL24 on 60S [13] (Figure 3c), we performed immunoblotting on all 60S-containing polysome fractions to evaluate the impact of RPL24 knockdown on eIF6 retention. Probing fractions corresponding to the area of the polysome profile near the 60S peak for Rack1, an obligatory 40S component, and RPL4, an obligatory $60 \mathrm{~S}$ component, confirmed that these fractions do, in fact, encompass the 60 subunit. As expected, RPL24 levels were drastically decreased in SKBR3 cells expressing RPL24 shRNA. Associated with RPL24 knockdown was a striking increase in 60S-associated eIF6 (Figure 3d). To rule out the possibility that the observed $60 \mathrm{~S}$ retention of eIF6 might be a false-positive or non-specific artifact of lentiviral expressed RPL24 shRNA, we overexpressed a functionally deficient truncation mutant of RPL24 that eliminates the last 17 amino acids. Polysome profiling of 293T cells expressing intact versus truncated RPL24 protein confirmed that truncated RPL24 can induce $60 \mathrm{~S}$ retention of eIF6 (Figure S1).

\section{Ribosomal protein acetylation is induced by pan- HDACi and HDAC6-selective inhibitors}

Previous studies have shown that pan-inhibitors of class I and II histone deacetylases (pan-HDACi), like TSA, can rapidly destabilize a number of oncogenic transcripts including HER2 in a cycloheximide-dependent manner (Figure S2, [25, 36]). Since cycloheximide inhibits polysome formation, these results suggested that polysomes are possibly involved in HER2 mRNA decay. Thus, SKBR3 cells were treated with TSA to evaluate polysome protein acetylation and determine if, similar to RPL24 depletion, HDACi can affect ribosome assembly dynamics. To detect early $(2 \mathrm{~h})$ ribosome or polysome acetylation following HDACi treatment ( $1 \mu \mathrm{M}$ TSA), SKBR3 polysomes were isolated using a discontinuous sucrose gradient as previously described [28]. Western blots using an antibody against acetylated lysine residues showed several TSA-induced bands, including a prominent TSA-induced acetyl-lysine protein band comigrating with RPL24 (Figure 4a, indicated by arrow), while total RPL24 levels were not altered by TSA. Mass spectrometry studies indicate that 15 ribosomal proteins, 11 large subunit proteins (RPL24 included) and 4 small subunit proteins, underwent at least a two-fold induction in acetylation following $2 \mathrm{~h}$ or $6 \mathrm{~h}$ TSA treatment $(1 \mu \mathrm{M})$ (Figure 4b, Figure S3a-q).

Like TSA, the HDAC6 (class IIb)-selective

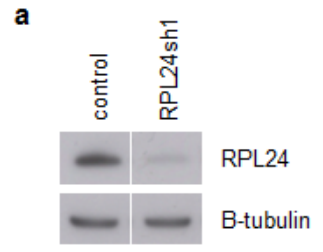

C

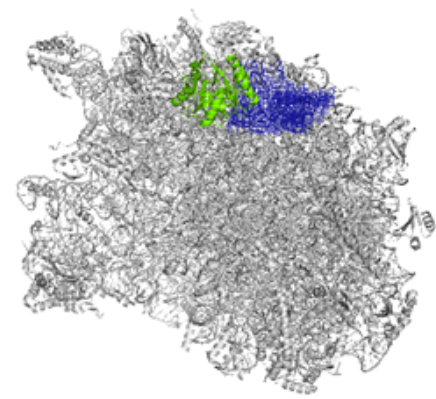

b

d

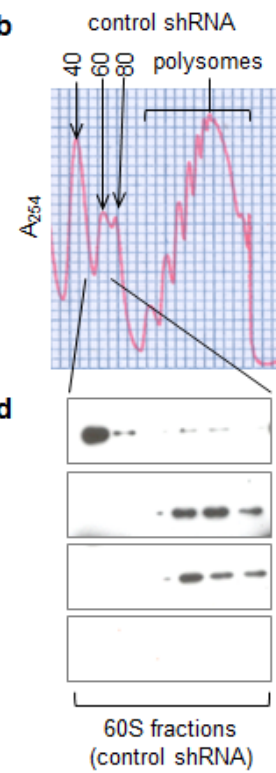

RPL24 shRNA

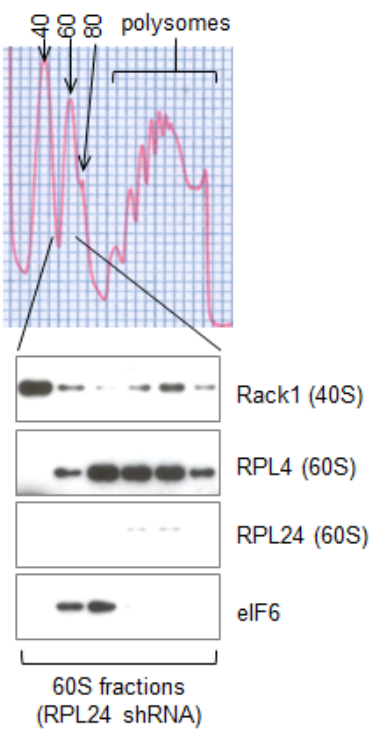

Figure 3: RPL24 knockdown reduces $80 \mathrm{~S}$ and polysome assembly while increasing 60S retention of eIF6. (a,b,d) SKBR3 cells were infected with lentiviruses expressing a GFP control or RPL24-targeting shRNA for three days. (a) Western blots using the indicated antibodies were performed on total cell lysates to assess knockdown efficiency. (b) Lysates were applied to a continuous sucrose gradient (10-50\%) and ultracentrifugation followed by fractionation was performed to separate ribosomal subunits and polysomes. (c) Pymol software was used to visualize the location of RPL24 (blue) relative to eIF6 (green) on the previously published structure of the 60S subunit in complex with eIF6 [13]. (d) Western blots using the indicated antibodies were performed on fractions from the 60S peaks using the indicated antibodies. 
inhibitors, Tubacin and ACY-775, as well as HDAC6 siRNA, all induce tubulin acetylation as expected as well as ribosomal protein acetylation, including the band that co-migrates with RPL24 (Figure 4c,d, indicated by arrows). Although the class I-specific HDACi, Entinostat, induces histone $\mathrm{H} 2 \mathrm{~B}$ acetylation without acetylating tubulin, it does not alter ribosomal protein acetylation even at a dose of $20 \mu \mathrm{M}$ (Figure 4c). Thus, the tubulinacetylating effects of pan-HDACi, known to be mediated by inhibition or knockdown of HDAC6, correspond to the observed ribosome and RPL24 acetylation responses induced by TSA.

Like RPL24 knockdown, HDACi reduces 80S assembly while increasing $60 \mathrm{~S}$ retention of eIF6 and reduces expression of cap (eIF4E)dependently translated proteins

Using continuous sucrose gradient fractionation of SKBR3 polysomes, $2 \mathrm{~h}$ culture treatment with TSA reduced $80 \mathrm{~S}$ and polysome assembly (Figure 5a) while
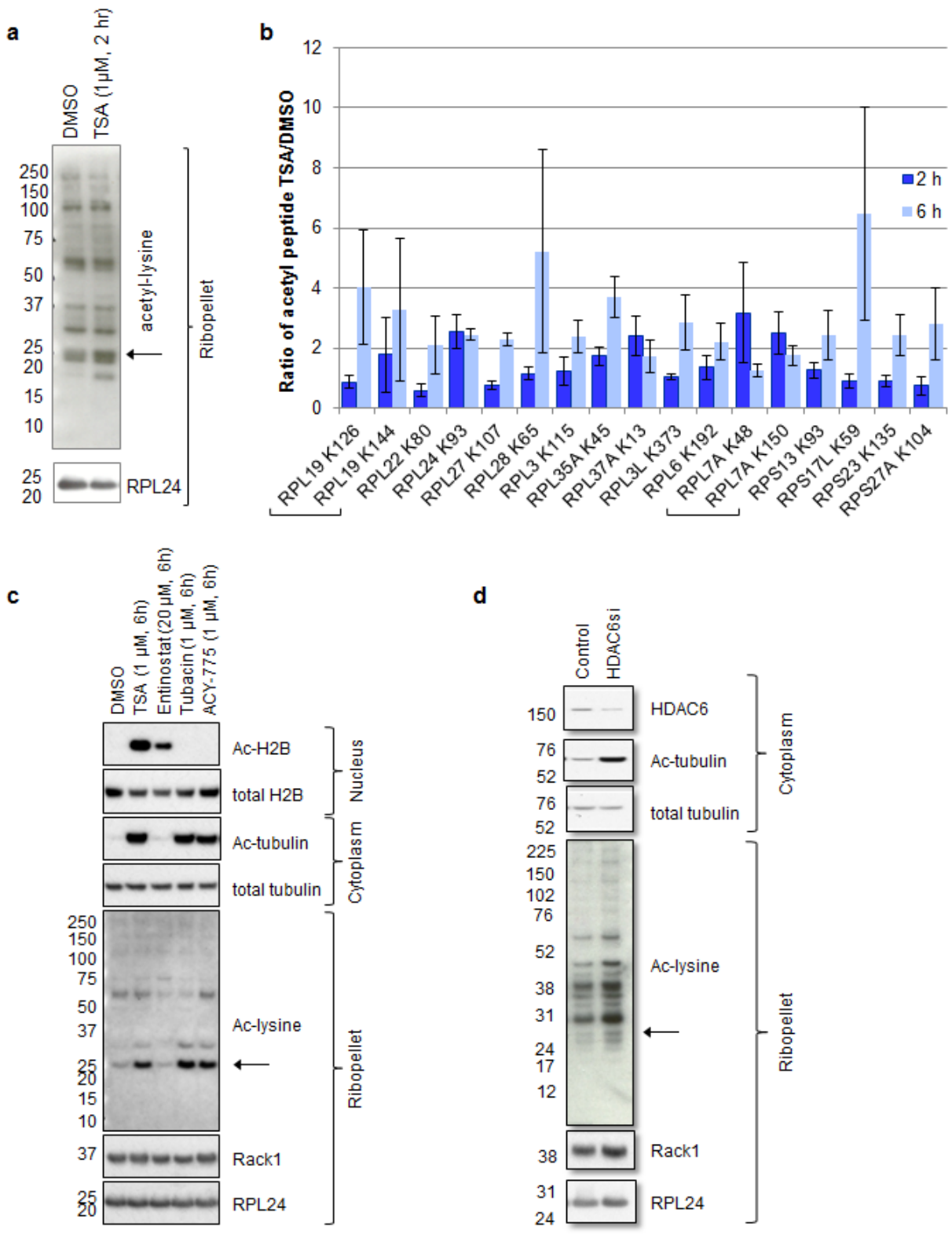

Figure 4: Ribosomal protein acetylation is induced by pan-HDACi and HDAC6-selective inhibitors. (a-c) SKBR3 cells were treated with the indicated drugs for the indicated period of time. (d) SKBR3 cells were transfected with the indicated siRNAs and allowed to incubate for 72 hours. (a, c, d). The indicated western blots were performed in ribopellets, total cytoplasmic lysates, or nuclear extracts. (b) Mass spectrometry was performed on ribopellets as described in materials and methods and in Figure 6 . The fold change in acetylated peptide to total peptide caused by TSA treatment is plotted. Only proteins that underwent at least a two-fold induction upon TSA treatment are shown. Error bars represent the standard error of the mean for three biological replicates. 
increasing $60 \mathrm{~S}$ retention of eIF6 without reducing $60 \mathrm{~S}$ RPL24 levels (Figure 5b). This result is comparable to that produced by RPL24 knockdown (Figure 3) or truncation (Figure S1). Furthermore, similar to RPL24

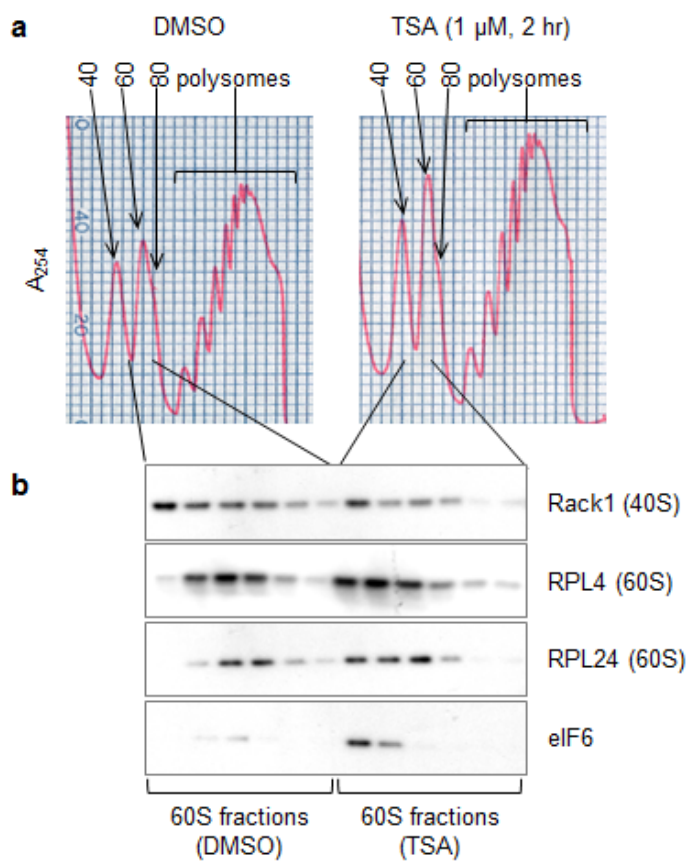

C

\begin{tabular}{|c|c|c|}
\hline $8 \mathrm{hr}$ & $24 \mathrm{hr}$ & \\
\hline ○ & ○ & TSA (nM) \\
\hline$--\cdots$ & -- & Cyclin D1 \\
\hline 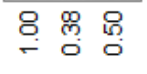 & 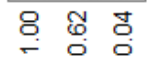 & Cyclin D1 / $\beta$-tubulin \\
\hline 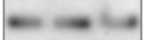 & $-\infty-\infty$ & Survivin \\
\hline$\stackrel{8}{\circ} \stackrel{\circ}{\leftarrow} \stackrel{8}{-}$ & 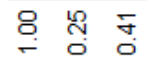 & Survivin / $\beta$-tubulin \\
\hline--- & $-\cdots$ & NBS1 \\
\hline$\stackrel{8}{\circ} \stackrel{\circ}{\circ} \stackrel{\circ}{\circ}$ & $\stackrel{8}{8} \stackrel{8}{\circ} \stackrel{5}{\circ}$ & NBS1 / $\beta$-tubulin \\
\hline$=0$ & 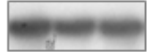 & GAPDH \\
\hline \& & 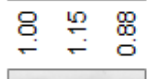 & GAPDH / $\beta$-tubulin \\
\hline$-\infty$ & $-\infty-\infty$ & $\beta$-tubulin \\
\hline
\end{tabular}

Figure 5: Like RPL24 knockdown, HDACi reduces $80 \mathrm{~S}$ assembly while increasing $60 \mathrm{~S}$ retention of eIF6 and reduces expression of cap (eIF4)-dependently translated proteins. $(a, b)$ SKBR3 cells were treated with TSA $(1 \mu \mathrm{M}, 2 \mathrm{~h})$. (a) Polysome profiles were carried out as previously described. (b) Western blots using the indicated antibodies were performed in fractions representing the 60S subunits. (c) SKBR3 cells were treated with TSA for the indicated doses and times, and proteins were identified by western blotting as indicated. Ratios of protein expression normalized to beta-tubulin levels were obtained using ImageJ software. knockdown, $24 \mathrm{~h}$ TSA treatment reduced the expression of the cap(eIF4e)-dependently translated proteins cyclin D1, survivin, and NBS1 relative to the housekeeping proteins GAPDH and $\beta$-tubulin (Figure $5 \mathrm{c}$ ). Shorter $(8 \mathrm{~h}$ ) TSA treatment reduced cyclin D1 levels but not survivin or NBS1 levels. The more rapid reduction of cyclin D1 levels was likely caused by the known effects of TSA on cyclin D1 transcription and transcript stability in addition to its effects on translation [37].

\section{HDACi enhances lysine (K27) acetylation on 60S RPL24}

Mass spectrometry studies were performed to identify sites of lysine (K) acetylation within RPL24 induced by HDACi. Continuous and discontinuous sucrose gradient fractionations were performed to isolate $60 \mathrm{~S}$ subunits and total polysomes respectively. Polyacrylamide gel electrophoresis was then performed on 60S and polysome samples and RPL24-containing bands were excised, trypsin digested, and subjected to mass spectrometry (LC-MS/MS) (Figure 6a). Among several detected acetylated RPL24 peptides, two were increased by TSA treatment; TDGKacVFQFLNAK (acetyl-K27) and AITGASLADIM*AKacR (acetyl-K93), where the internal lysines in both peptides are $\mathrm{N}$-acetylated (Kac). As the MS experiments of the 60S polysome were performed after in-gel digestion the methionine residue of the second peptide was predominantly oxidized $\left(\mathrm{M}^{*}=\mathrm{M}+16\right)$, as commonly observed during SDS PAGE processing. In independent, in-solution digestion experiments, we also identified the corresponding non-oxidized form of acetylated peptide AITGASLADIMAKacR with correlating MS/MS fragmentation pattern. Representative spectra are shown for TDGKac VFQFLNAK (acetyl-K27) and AITGASLADIM*AKacR (acetyl-K93)) (Figure S4a,b). In 3 biological replicate experiments, the amount of K27-acetylated RPL24, normalized to total protein concentration within the $60 \mathrm{~S}$ subunit, was increased at least 2-fold within $2 \mathrm{~h}$ of TSA treatment. However, there was no significant induction of RPL24 K27 acetylation found within polysomes (not containing 60S subunits) (Figure 6b). In contrast, RPL24 K93 acetylation within the $60 \mathrm{~S}$ subunits was not significantly changed by TSA treatment, yet K93 acetylation was induced 2.5-fold within RPL24 associated with polysomes (Figure 6c). Given the proximity of the T. thermophilia RPL24 K26 site (that resides in a homologous region to human K27) to eIF6 (Figure 7a), these findings implicate involvement of the TSA induced acetylation of RPL24 at K27 in preventing $40 \mathrm{~S}-60 \mathrm{~S}$ subunit joining and $60 \mathrm{~S}$ retention of eIF6.

\section{DISCUSSION}

Consistent with the role of RPL24 in protecting 
against Akt-driven and Myc-driven murine tumorigenesis $[7,8]$, the present study provides new evidence that, not only is elevated RPL24 expression common in human breast tumors, but depletion or structural alteration of RPL24 can significantly impair human breast cancer cell viability (Figure 1,2). Our results indicate that this could occur through a reduction in cap(eIF4E)dependent translation of proteins essential for cancer cell proliferation (e.g. cyclin D1), survival (e.g. survivin) and genome repair (e.g. NBS1) (Figure 2). Our findings also indicate that the mechanism of action by which RPL24 deficiency reduces expression of these proteins essential
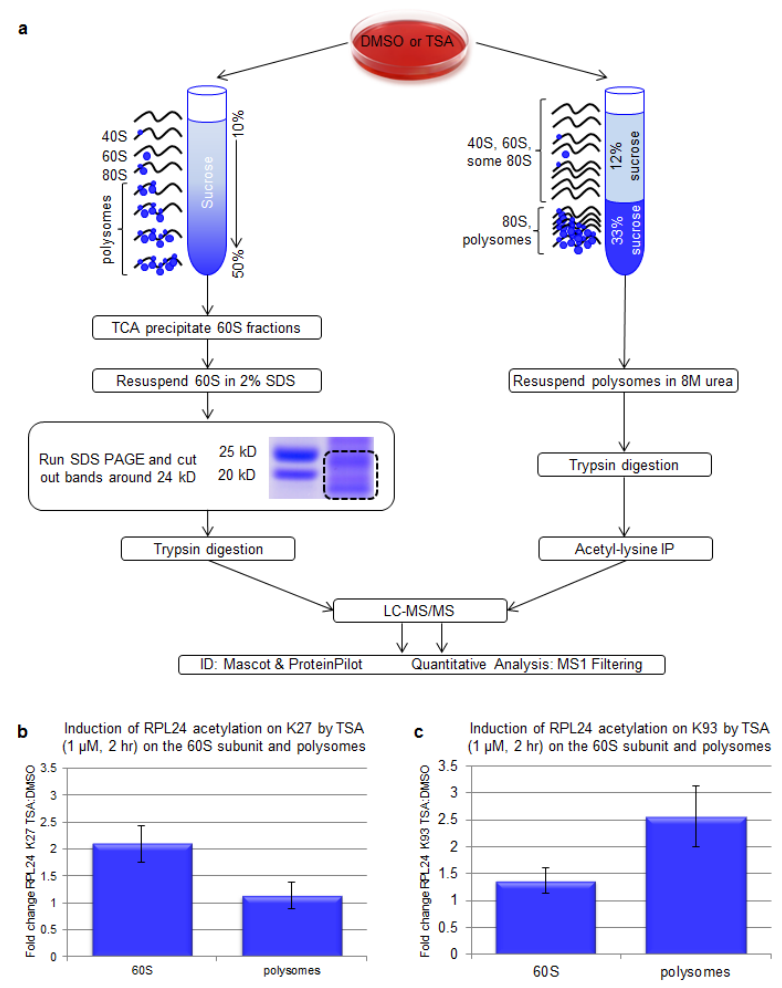

Figure 6: HDACi enhances lysine (K27) acetylation on 60S, but not polysomal, RPL24. (a) Schematic of mass-spectrometry-based techniques to analyze ribosomal protein acetylation. SKBR3 cells were treated with TSA (1 $\mu \mathrm{M}, 2 \mathrm{~h}$ or $6 \mathrm{~h}$ ). To isolate $60 \mathrm{~S}$ subunits, polysome profiles were performed and $60 \mathrm{~S}$ fractions were TCA precipitated. Concentrated $60 \mathrm{~S}$ samples were resolved on $4-12 \%$ bis-tris gels and RPL24-containing bands were excised and trypsin digested. In parallel, polysomes were isolated using a discontinuous sucrose gradient as described. Trypsin digestions and acetyl lysine immunoprecipitations were subsequently carried out. Mass spectrometry was performed on 60S-associated RPL24containing gel bands or polysome-containing acetyl-lysine immunoprecipitations as described in the methods section. (b,c) On 60S-associated and polysome-associated RPL24, the fold induction caused by TSA ( $1 \mu \mathrm{M}, 2$ h) of K27 (b) or K93 (c)acetylated peptide (normalized to total protein concentration) was plotted. Error bars represent the standard error of the mean for three biological replicates. Note: the data for K93 acetylation of RPL24 K93 is also shown in Figure 4b. for cancer cell viability involves failure of $40 \mathrm{~S}$ and $60 \mathrm{~S}$ ribosomal subunit joining into functional $80 \mathrm{~S}$ ribosomes by retention of eIF6 on 60S subunits (Figure 3).

Although RPL24 is an essential ribosomal protein, partial (up to $70 \%$ ) depletion of RPL24 does not reduce the expression of all proteins but preferentially inhibits expression of eIF4E-regulated and cap-dependently translated proteins. In particular, the translation of housekeeping genes like GAPDH and $\beta$-tubulin remained unaffected when assessed by protein levels normalized to total cell number (Figure 2). In contrast, cyclin D1, survivin, and NBS1 are among several well-known growth, survival and tumorigenic transcripts with highly structured 5' untranslated regions (UTRs) whose capdependent ribosomal translation is tightly regulated by eIF4E; and their synthesis was reduced by as much as $75 \%$ following partial depletion of RPL24. These results raise the possibility that RPL24 depletion changes ribosome structure or recruitment factors in a manner that causes ribosomes to be recruited to cap-dependently translated mRNAs less efficiently.

As demonstrated in this study and schematically illustrated in Figure 7b, treatment of breast cancer cells with a pan-HDACi like TSA induces recruitment of eIF6

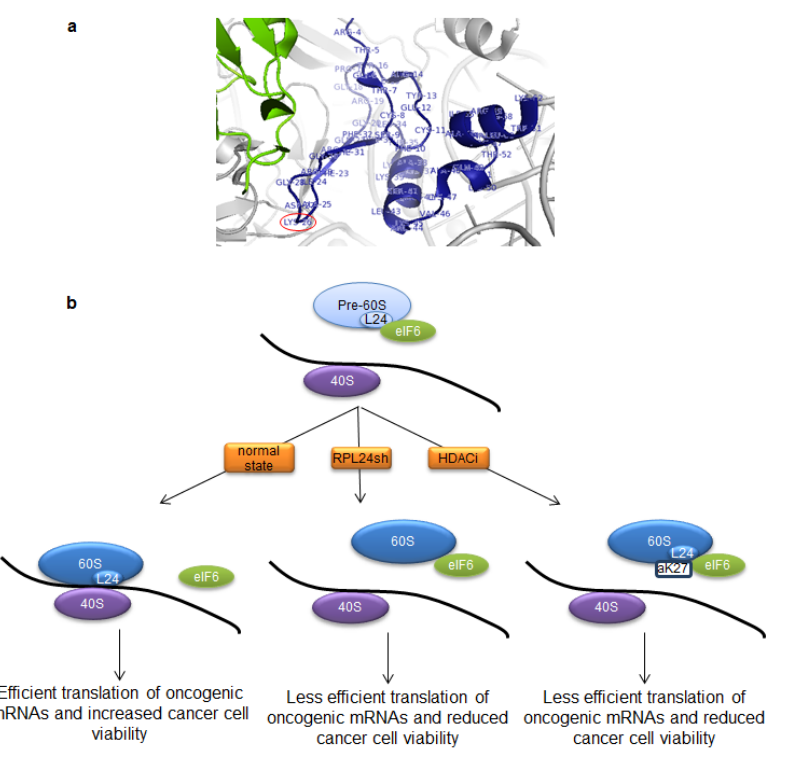

Figure 7: Schematic for modulation of ribosome assembly by RPL24 acetylation. (a) A magnified portion of the RPL24 (blue)-eIF6 (green) interface, visualized with Pymol software, from previous x-ray crystallography data [13], is shown (zoomed out view shown in Figure 3c). T. thermophilia RPL24 residues are labelled and K26, which resides in a region of RPL24 homologous to where human K27 resides, is circled. (b) eIF6 binds to the pre-60S near RPL24 to prevent premature association of the $40 \mathrm{~S}$ and $60 \mathrm{~S}$ ribosomal subunits; eIF6 is then released from the mature $60 \mathrm{~S}$, allowing it to join with the $40 \mathrm{~S}$ to form the $80 \mathrm{~S}$ ribosome. Our model indicates that either RPL24 depletion or TSA (HDACi)-induced RPL24 acetylation on K27 prevents eIF6 release and 80S formation. 
to 60 S subunits along with acetylation of RPL24 at K27, likely impairing 40S-60S joining. These phenotypes mimic RPL24 knockdown (Figure 3, Figure 5). Consistent with this finding, the crystal structure of the $T$. thermophilia 60S subunit [13] reveals that RPL24 K26, which closely aligns with human RPL24 K27, is adjacent to eIF6 (Figure 3c, 7a). While we detected multiple sites of acetylation on RPL24, only K27 acetylation was induced by HDACi in association with the 60S subunit. Similar to RPL24 depletion, $24 \mathrm{~h}$ of HDACi treatment reduced the expression of cyclin D1, survivin, and NBS1 relative to GAPDH and $\beta$-tubulin (Figure $5 \mathrm{c}$ ), a possible secondary consequence of the 60S acetylation and ribosome assembly failure noted as early as $2 \mathrm{~h}$ after TSA treatment. These timing differences in responses to the same TSA treatment suggest that although ribosome assembly is inhibited rapidly, it takes hours for the effects of such a functional impairment in ribosome assembly to manifest as a significant reduction in the pools of proteins synthesized by cap-dependent translation.

Our findings point toward the existence of an as yet unrecognized endogenous and acetylation-dependent mechanism that regulates both polysome assembly and cap-dependent protein translation. Including RPL24, we identified fifteen ribosomal proteins that undergo acetylation induction two-fold or more as a rapid consequence of HDACi treatment. These proteins are also potential contributors to this acetylation-dependent control of ribosome assembly. Like pan-HDACi treatment, enzymatic inhibition or knockdown of cytoplasmic HDAC6 appears to be sufficient to induce polysomal protein acetylation, in contrast to a class I-selective HDACi like Entinostat which has no such affect (Figure 4). Since only 2-6 h treatment with an HDAC6-selective inhibitor induces near maximum levels of ribosome protein acetylation, this posttranslational structural change is likely due to a direct cytoplasmic HDAC effect on ribosomal proteins rather than any indirect effect mediated by HDAC regulated nuclear gene expression.

In view of the current lack of any drug-like agent that can specifically target RPL24, our study also points toward RPL24 as a previously unrecognized subcellular target potentially contributing to the known anticancer activity of two approved cancer therapeutics, vorinostat and romidepsin, that are HDACi's like TSA, not to mention contributing to the anticancer mechanisms of investigational HDAC6-selective inhibitors like ACY-775. The fact that homozygous loss of RPL24 is embryonic lethal [6] might cause concern about developing a targetspecific anti-RPL24 cancer therapeutic. However, it is important to recall that the homozygous deletion of many well-established breast cancer drug targets, such as human epidermal growth factor receptor (HER2), mTOR, and the catalytic domain of PI3K, are also embryonic lethal [38-41], yet this fact did not preclude or even delay the successful clinical development of therapeutics selective for these targets. The fact that RPL24 haploinsufficiency is compatible with normal murine lifespan is additionally reassuring from the standpoint of developing an antiRPL24 therapeutic, and points to the established fact that all approved anticancer agents rely on in vivo definition of an optimal therapeutic window, whereby anticancer efficacy vastly exceeds host toxicity. Given that RPL24 haploinsufficiency protects mice from Akt- and Mycdriven tumorigenesis $[7,8]$, it is interesting to note that at least $40 \%$ of basal-like breast cancers overexpress Akt or Myc [42], indicating that anti-RPL24 therapeutics could be effective against basal-like and other clinically aggressive subsets of human breast cancer.

\section{METHODS}

\section{Analysis of RPL24 expression in patient-matched breast carcinoma and normal breast tissue}

Expression data from 43 patient-matched breast carcinoma and normal breast tissue samples assayed on Affymetrix U133A microarrays (GSE15852) was obtained from the Gene Expression Omnibus (GEO) [22]. Raw data was RMA-normalized, annotated using its associated platform annotation file (GPL96-39578) and meancentered. Expression levels of the RPL24 probe within the patient-matched tumor and normal samples were obtained and compared. Significance was assessed using the paired t-test.

\section{Cell culture}

SKBR3 human breast cancer cells (American Type Culture Collection (ATCC), Rockville, MD) were grown in McCoy's 5A media supplemented with $10 \%$ fetal bovine serum (FBS) and L-glutamate. 293T cells (American Type Culture Collection (ATCC), Rockville, MD) were grown in DMEM with 10\% FBS and L-glutamate.

\section{shRNA and retroviral infection}

Lentiviral vectors containing shRNAs toward RPL24, TRCN0000117642/RPL24sh1/target sequence CCTGAAGTTAGAAAGGCTCAA and TRCN0000117643/RPL24sh2/target sequence GTGCATCTCTTGCTGATATAA, and a green fluorescent protein control RHS4459/control/target sequence TACAACAGCCACAACGTCTAT were purchased from Thermo Scientific (formerly Open Biosystems, Cincinnati, OH). shRNA expressing lentiviruses were produced as previously described [23-25]. Briefly, 293T cells were transfected with lentiviral vectors along with packaging vectors. One day later, media was changed to 
Optimem (Life Technologies, Grand Island, NY) and the virus was collected for two days and concentrated it as outlined previously [26, 27]. SKBR3 cells were infected in the presence of $6 \mu \mathrm{g} / \mathrm{ml}$ polybrene with a multiplicity of infection of $\sim 2$. One day after infection media was changed to regular growth media in the case of transient infections or growth media with $0.5 \mu \mathrm{g} / \mathrm{ml}$ puromycin in the case of stable transfections.

\section{siRNA transfection}

The following siRNAs were purchased from (Thermo Scientific-Dharmacon, Chicago, IL): HDAC6targeting smart pool (L-003499-00) and non-targeting control pool (D-001810-10-05). Lipofectamine 2000 (Life Technologies) was used to transfect SKBR3 cells per manufacturer's protocol. Cells were analyzed 72 hours after transfection.

\section{Viability assay}

Cells infected with different shRNA-expressing lentiviruses were plated in 96-well plates at a density of 5,000 cells per well. Three hours after plating $\left(\mathrm{T}_{0}\right)$, a base line viability reading was taken using the CellTiter-Glo Luminescent Cell Viability Assay (Promega, Madison, WI). Four days later $\left(\mathrm{T}_{4}\right)$ another reading was taken using the same assay. For each treatment, each of three $\mathrm{T}_{4}$ data points was divided by the average of all three $\mathrm{T}_{0}$ data points for that treatment. The data from the RPL24 shRNA-treated cells was then normalized to that from the control cells. Data is represented by the mean and standard deviation of triplicates.

\section{Cell lysis and western blotting}

Cells were lysed in RIPA buffer (10 mM Tris-HCL (pH 8.0), 1mM EDTA, 0.5 mM EGTA, 1\% triton X-100, $0.1 \%$ sodium deoxycholate, $0.1 \% \mathrm{SDS}, 140 \mathrm{mM} \mathrm{NaCl}, 20$ $\mathrm{mM} \mathrm{NaF}$, Complete EDTA-free protease inhibitor tablets (Roche Diagnostics Corp., Basel, Switzerland) and the phosphatase inhibitor cocktail PhosSTOP (Roche)), the latter two as indicated by the manufacturer's protocol. Equal amounts of protein were diluted in $2 \mathrm{X}$ sample buffer. Immunoblots on PVDF (Polyvinyldene Fluoride) membranes were blocked with nonfat milk in tris-buffered saline with $0.05 \%$ tween-20 (TBST). The following antibodies were incubated with membranes in 5\% nonfat milk in TBST: RPL24 (Proteintech, Chicago, IL), Cyclin D1, Rack1, RPL4 (Santa Cruz Biotechnology, Santa Cruz, CA), NBS1, GAPDH (EMD Millipore Corporation, Chicago, IL), Survivin, $\beta$-tubulin, acetyl-lysine, eIF6, acetyl-H2B, H2B, (Cell Signaling Technology, Boston, MA), acetyl-tubulin, tubulin (Sigma Aldrich (St. Louis,
MO)). Densitometry was performed using ImageJ software.

\section{Isolation of ribosomes}

Cells, plated at $\sim 90 \%$ confluency, were treated as indicated. After treatment, cells were treated with $50 \mu \mathrm{g} /$ $\mathrm{ml}$ cycloheximide for 15 minutes. Cells were lysed with a buffer containing $10 \mathrm{mM}$ HEPES, $10 \mathrm{mM} \mathrm{KCl,} 75 \mathrm{mM}$ $\mathrm{NaCl}, 10 \mathrm{mM} \mathrm{MgCl}, 0.35 \%$ NP40, pH 7.9 supplemented with Complete EDTA-free protease inhibitor tablets, PhosSTOP phosphatase inhibitor tablets (Roche) per manufacturer's instructions, $50 \mu \mathrm{g} / \mathrm{ml}$ cycloheximide, SUPERase RNase inhibitors (Life Technologies) per manufacturer's instructions, $15 \mu \mathrm{M}$ TSA, and $5 \mathrm{mM}$ nicotinimide to inhibit HDACs. Supernatants were collected as cytoplasmic preparations. Where indicated, pellets containing nuclei were resuspended in RIPA buffer (described above). The suspension was spun at 13,000 rpm for $5 \mathrm{~min}$ and supernatants were collected as nuclear preparations.

Ribosomes were subsequently isolated from cytoplasmic preparations as described previously [28]. Briefly, lysates were layered on top of a $12 \%$ and $33 \%$ discontinuous sucrose gradient and spun at 38,000 rpm for $2 \mathrm{~h}$. The resulting polysome pellet was then resuspended, stripped of RNA with acetic acid, and then pelleted with acetone. The pellet was then resuspended in $8 \mathrm{M}$ urea, $2 \%$ CHAPS, and $25 \mathrm{mM}$ dithiothreitol (DTT).

Polysome profiles to separate the $40 \mathrm{~S}, 60 \mathrm{~S}, 80 \mathrm{~S}$ and polysomes were carried out by layering cell lysates over a continuous 10-50\% sucrose gradient and spun at 38,000 rpm for $2 \mathrm{~h}$ as previously described [29, 30]. Fractions were collected using a Retriever 500 fraction collector with a UV (UA6) detector (ISCO Teledyne (Lincoln, Nebraska)).

\section{Visualization of crystallography data}

Pymol software (Schrodinger, Mannheim, Germany) was used to visualize RPL24 and eIF6 on previously published crystallography data of the Tetrahymena thermophilia 60S ribosomal subunit (human gene names used) bound to eIF6 [13].

\section{Drugs}

TSA was obtained from Sigma Aldrich, Entinostat from Syndax (Walthan, MA) and Tubacin (Caymen Chemicals, Ann Arbor, MI). ACY-775 was obtained from Acetylon Pharmaceuticals (Boston, MA). 


\section{Mass spectrometry}

To prepare polysome samples for mass spectrometry, cells were treated with HDACi and polysome pellets were prepared using a discontinuous sucrose gradient as described above. Protein concentration was determined using the Pierce ${ }^{\mathrm{TM}}$ BCA Protein Assay Kit (Thermo Scientific) and equal amount of protein were trypsin digested. Acetyl-lysine immunoprecipitations were carried on resultant peptides out using an antibody from Cell Signaling Technology. Acetyl-proteins were then eluted, extracted, and desalted.

To determine the acetylation status of 60S subunit proteins, cells were treated with HDACi and polysome profiles were performed as described above. The four fractions representing the 60S subunit were identified, via western blots, for the absence of Rack1 (40S marker) and the presence of RPL24 (60S marker). Those 60S fractions were then TSA precipitated and reconstituted in $2 \%$ SDS. 60 S samples (normalized by protein loading) were resolved using 4-12\% Bis-Tris gels and stained with Imperial Protein Stain (Thermo). Gel bands were excised, diced into small pieces, destained, reduced with $10 \mathrm{mM}$ dithiothreitol, and alkylated with $5 \mathrm{mM}$ iodoacetamide. Ingel trypsin digestion was performed using a 1:20 enzyme to protein ratio for $16 \mathrm{~h}$ at $37^{\circ} \mathrm{C}$. Resultant peptides were extracted and desalted.

Three biological replicates of polysome or $60 \mathrm{~S}$ samples were then analyzed by LC-MS/MS using a quadrupole time-of-flight (QqTOF)TripleTOF 5600 mass spectrometer (AB SCIEX, Dublin, CA) coupled to an Eksigent (Dublin, CA) nanoLC Ultra, 2D plus. Briefly, the resulting peptides were chromatographically separated on a C-18 reversed-phase analytical column $(75 \mu \mathrm{m}$ I.D.) connected to the TripleTOF 5600 operating in data dependent mode (1 MS1 survey scan followed by $30 \mathrm{MS} /$ MS scans per 1.8 second acquisition cycle) [31]. Mascot v2.3.02 and ProteinPilot v4.5 data base searches were employed for peptide identification (Table S1, Table S2) using a false discovery rate analysis (FDR) of 0.01. For MS/MS spectral data of acetylated peptides see Figures S3 and S4. Moreover, more detailed interactive viewing of spectral libraries is available at the Panorama webserver (University of Washington, Seattle), at 'https://daily. panoramaweb.org/labkey/project/Gibson/Polysomes Benz2/begin.view?'.

Quantitative data analysis of acetyl-lysine peptides was performed by integration of selected molecular ion intensities using Skyline MS1 Filtering as previously described [31]. The average signal intensity, as determined by the area under the curve (AUC) of the LC chromatogram, of the replicate biological samples was calculated. The amount of acetylated peptide normalized to total protein loaded onto the gel for each condition was determined and the fold induction upon TSA treatment was then calculated.

\section{CONCLUSIONS}

This is the first study implicating a role for RPL24 expression in human breast tumors and in regulating protein translation necessary for breast tumorigenesis. We demonstrate that RPL24 depletion decreases the level of proteins synthesized by cap-dependent translation essential for cancer cell growth, survival and genome repair, without altering the synthesis of housekeeping proteins. Polysome profiling indicates that this partial translation defect might be related to the control of eIF6 release from the $60 \mathrm{~S}$ subunit, inhibiting assembly of $80 \mathrm{~S}$ ribosomes and polysomes. Furthermore, we show that HDACi and more specifically, inhibition of HDAC6, rapidly induces acetylation of multiple ribosomal proteins, and that RPL24 acetylation at K27 is associated with impairment in ribosome assembly similarly induced by RPL24 depletion. Therefore, our findings point toward the existence of an endogenous, HDAC6- and protein deacetylation-dependent polysome assembly mechanism that can regulate oncogenic growth and potentially serve as a novel cancer therapeutic target.

\section{Competing Interests}

The authors declare no conflicts of interest.

\section{Author's Contributions}

Conceived and designed the experiments: KAW, AK, GKS, JMH, BWG, CCB. Performed experiments: KAW, AK, GKS, DER, BSG, MAY, IMH. Analyzed and interpreted data: KAW, AK, GKS, CY, BS, JMH, BWG, CCB. Wrote the paper: KAW, AK, BS, CCB.

\section{ACKNOWLEDGEMENTS}

This research was supported by NIH/NCI grants R21-CA155679, R01-CA071468, and U24-CA14358, NIH/NIA T32-AG000266, and by Hazel P. Munroe memorial funding to the Buck Institute. This work was also supported by an NCRR shared instrumentation grant for the TripleTOF 5600 (1S10 OD016281; BWG). We appreciate the genorisity of Syndax (Walthan, MA) for supplying Entinostat(MS-275) and Acetylon Pharmaceuticals(Boston, MA) for supplying ACY-775. We thank Aric Rogers for advice regarding polysome profiling. We are also grateful to members of the Benz lab for insightful discussions.

\section{REFERENCES}

1. Mayer C, Grummt I: Ribosome biogenesis and cell growth: mTOR coordinates transcription by all three classes of 
nuclear RNA polymerases. Oncogene 2006, 25(48):63846391.

2. Thoreen CC, Chantranupong L, Keys HR, Wang T, Gray NS, Sabatini DM: A unifying model for mTORC1mediated regulation of mRNA translation. Nature 2012, 485(7396):109-113.

3. Hsu PP, Kang SA, Rameseder J, Zhang Y, Ottina KA, Lim D, Peterson TR, Choi Y, Gray NS, Yaffe MB et al: The mTOR-regulated phosphoproteome reveals a mechanism of mTORC1-mediated inhibition of growth factor signaling. Science 2011, 332(6035):1317-1322.

4. Hsieh AC, Liu Y, Edlind MP, Ingolia NT, Janes MR, Sher A, Shi EY, Stumpf CR, Christensen C, Bonham MJ et al: The translational landscape of mTOR signalling steers cancer initiation and metastasis. Nature 2012, 485(7396):55-61

5. Ounap K, Kasper L, Kurg A, Kurg R: The Human WBSCR22 Protein Is Involved in the Biogenesis of the 40S Ribosomal Subunits in Mammalian Cells. PloS one 2013, 8(9):e75686.

6. Oliver ER, Saunders TL, Tarle SA, Glaser T: Ribosomal protein L24 defect in belly spot and tail (Bst), a mouse Minute. Development 2004, 131(16):3907-3920.

7. Hsieh AC, Costa M, Zollo O, Davis C, Feldman ME, Testa JR, Meyuhas O, Shokat KM, Ruggero D: Genetic dissection of the oncogenic mTOR pathway reveals druggable addiction to translational control via 4EBP-eIF4E. Cancer cell 2010, 17(3):249-261.

8. Barna M, Pusic A, Zollo O, Costa M, Kondrashov N, Rego E, Rao PH, Ruggero D: Suppression of Myc oncogenic activity by ribosomal protein haploinsufficiency. Nature 2008, 456(7224):971-975.

9. Barkic M, Crnomarkovic S, Grabusic K, Bogetic I, Panic L, Tamarut S, Cokaric M, Jeric I, Vidak S, Volarevic S: The p53 tumor suppressor causes congenital malformations in Rpl24-deficient mice and promotes their survival. Molecular and cellular biology 2009, 29(10):2489-2504.

10. Dresios J, Derkatch IL, Liebman SW, Synetos D: Yeast Ribosomal Protein L24 Affects the Kinetics of Protein Synthesis and Ribosomal Protein L39 Improves Translational Accuracy, While Mutants Lacking Both Remain Viable†. Biochemistry 2000, 39(24):7236-7244.

11. Kruiswijk T, Planta RJ, Krop JM: The course of the assembly of ribosomal subunits in yeast. Biochimica et biophysica acta 1978, 517(2):378-389.

12. Saveanu C, Namane A, Gleizes PE, Lebreton A, Rousselle JC, Noaillac-Depeyre J, Gas N, Jacquier A, FromontRacine M: Sequential protein association with nascent $60 \mathrm{~S}$ ribosomal particles. Molecular and cellular biology 2003, 23(13):4449-4460.

13. Klinge $\mathrm{S}$, Voigts-Hoffmann F, Leibundgut $\mathrm{M}$, Arpagaus $\mathrm{S}$, Ban N: Crystal structure of the eukaryotic 60S ribosomal subunit in complex with initiation factor 6. Science 2011, 334(6058):941-948.
14. Klinge S, Voigts-Hoffmann F, Leibundgut M, Ban N: Atomic structures of the eukaryotic ribosome. Trends in biochemical sciences 2012, 37(5):189-198.

15. Gartmann M, Blau M, Armache JP, Mielke T, Topf M, Beckmann R: Mechanism of eIF6-mediated inhibition of ribosomal subunit joining. The Journal of biological chemistry 2010, 285(20):14848-14851.

16. Ceci M, Gaviraghi C, Gorrini C, Sala LA, Offenhauser N, Marchisio PC, Biffo S: Release of eIF6 (p27BBP) from the 60S subunit allows $80 \mathrm{~S}$ ribosome assembly. Nature 2003, 426(6966):579-584.

17. Finch AJ, Hilcenko C, Basse N, Drynan LF, Goyenechea B, Menne TF, Gonzalez Fernandez A, Simpson P, D’Santos $\mathrm{CS}$, Arends MJ et al: Uncoupling of GTP hydrolysis from eIF6 release on the ribosome causes Shwachman-Diamond syndrome. Genes \& development 2011, 25(9):917-929.

18. Brina D, Grosso S, Miluzio A, Biffo S: Translational control by $80 \mathrm{~S}$ formation and $60 \mathrm{~S}$ availability: the central role of eIF6, a rate limiting factor in cell cycle progression and tumorigenesis. Cell Cycle 2011, 10(20):3441-3446.

19. Miluzio A, Beugnet A, Volta V, Biffo S: Eukaryotic initiation factor 6 mediates a continuum between $60 \mathrm{~S}$ ribosome biogenesis and translation. EMBO reports 2009, 10(5):459-465.

20. Gandin V, Miluzio A, Barbieri AM, Beugnet A, Kiyokawa $\mathrm{H}$, Marchisio PC, Biffo S: Eukaryotic initiation factor 6 is rate-limiting in translation, growth and transformation. Nature 2008, 455(7213):684-688.

21. Kentsis A, Topisirovic I, Culjkovic B, Shao L, Borden KL: Ribavirin suppresses eIF4E-mediated oncogenic transformation by physical mimicry of the 7-methyl guanosine mRNA cap. Proceedings of the National Academy of Sciences of the United States of America 2004, 101(52):18105-18110.

22. Pau Ni IB, Zakaria Z, Muhammad R, Abdullah N, Ibrahim N, Aina Emran N, Hisham Abdullah N, Syed Hussain SN: Gene expression patterns distinguish breast carcinomas from normal breast tissues: the Malaysian context. Pathology, research and practice 2010, 206(4):223-228.

23. Freund A, Patil CK, Campisi J: p38MAPK is a novel DNA damage response-independent regulator of the senescenceassociated secretory phenotype. EMBO J 2011, 30(8):15361548 .

24. Laberge R-M, Zhou L, Sarantos MR, Rodier F, Freund A, de Keizer PLJ, Liu S, Demaria M, Cong Y-S, Kapahi $P$ et al: Glucocorticoids suppress selected components of the senescence-associated secretory phenotype. Aging Cell 2012, 11(4):569-578.

25. Scott GK, Marden C, Xu F, Kirk L, Benz CC: Transcriptional repression of ErbB2 by histone deacetylase inhibitors detected by a genomically integrated ErbB2 promoter-reporting cell screen. Mol Cancer Ther 2002, 1(6):385-392.

26. Wilson KA, Colavito SA, Schulz V, Wakefield PH, Sessa 
W, Tuck D, Stern DF: NFBD1/MDC1 regulates Cav1 and Cav2 independently of DNA damage and p53. Mol Cancer Res 2011, 9(6):766-781.

27. Wilson KA, Stern DF: NFBD1/MDC1, 53BP1 and BRCA1 have both redundant and unique roles in the ATM pathway. Cell Cycle 2008, 7(22):3584-3594.

28. Barritault D, Expert-Bezancon A, Guerin MF, Hayes D: The use of acetone precipitation in the isolation of ribosomal proteins. Eur J Biochem 1976, 63(1):131-135.

29. Rogers Aric N, Chen D, McColl G, Czerwieniec G, Felkey K, Gibson Bradford W, Hubbard A, Melov S, Lithgow Gordon J, Kapahi P: Life Span Extension via eIF4G Inhibition Is Mediated by Posttranscriptional Remodeling of Stress Response Gene Expression in C. elegans. Cell Metabolism 2011, 14(1):55-66.

30. Wilson-Edell KA, Yevtushenko MA, Rothschild DE, Rogers AN, Benz CC: mTORC1/C2 and pan-HDAC inhibitors synergistically impair breast cancer growth by convergent AKT and polysome inhibiting mechanisms. Breast cancer research and treatment 2014, 144(2):287-298.

31. Schilling B, Rardin MJ, MacLean BX, Zawadzka AM, Frewen BE, Cusack MP, Sorensen DJ, Bereman MS, Jing E, Wu CC et al: Platform-independent and label-free quantitation of proteomic data using MS1 extracted ion chromatograms in skyline: application to protein acetylation and phosphorylation. Mol Cell Proteomics 2012, 11(5):202214.

32. Ni IB, Ching NC, Meng CK, Zakaria Z: Translocation $\mathrm{t}(11 ; 14)(\mathrm{q} 13 ; \mathrm{q} 32)$ and genomic imbalances in multi-ethnic multiple myeloma patients: a Malaysian study. Hematology reports 2012, 4(3):e19.

33. Pettersson F, Yau C, Dobocan MC, Culjkovic-Kraljacic B, Retrouvey H, Puckett R, Flores LM, Krop IE, Rousseau $\mathrm{C}$, Cocolakis E et al: Ribavirin treatment effects on breast cancers overexpressing eIF4E, a biomarker with prognostic specificity for luminal B-type breast cancer. Clinical cancer research : an official journal of the American Association for Cancer Research 2011, 17(9):2874-2884.

34. Sonenberg N: eIF4E, the mRNA cap-binding protein: from basic discovery to translational research. Biochemistry and cell biology $=$ Biochimie et biologie cellulaire 2008, 86(2):178-183.

35. Ye Q, Cai W, Zheng Y, Evers BM, She QB: ERK and AKT signaling cooperate to translationally regulate survivin expression for metastatic progression of colorectal cancer. Oncogene 2013.

36. Scott GK, Marx C, Berger CE, Saunders LR, Verdin E, Schafer S, Jung M, Benz CC: Destabilization of ERBB2 transcripts by targeting 3' untranslated region messenger RNA associated HuR and histone deacetylase-6. Mol Cancer Res 2008, 6(7):1250-1258.

37. Alao JP, Lam EW, Ali S, Buluwela L, Bordogna W, Lockey P, Varshochi R, Stavropoulou AV, Coombes RC, Vigushin DM: Histone deacetylase inhibitor trichostatin A represses estrogen receptor alpha-dependent transcription and promotes proteasomal degradation of cyclin D1 in human breast carcinoma cell lines. Clinical cancer research : an official journal of the American Association for Cancer Research 2004, 10(23):8094-8104.

38. Lee K-F, Simon H, Chen H, Bates B, Hung M-C, Hauser $\mathrm{C}$ : Requirement for neuregulin receptor erbB2 in neural and cardiac development. Nature 1995, 378(6555):394-398.

39. Gangloff YG, Mueller M, Dann SG, Svoboda P, Sticker M, Spetz JF, Um SH, Brown EJ, Cereghini S, Thomas G et al: Disruption of the mouse mTOR gene leads to early postimplantation lethality and prohibits embryonic stem cell development. Molecular and cellular biology 2004, 24(21):9508-9516.

40. Murakami M, Ichisaka T, Maeda M, Oshiro N, Hara K, Edenhofer F, Kiyama H, Yonezawa K, Yamanaka S: mTOR is essential for growth and proliferation in early mouse embryos and embryonic stem cells. Molecular and cellular biology 2004, 24(15):6710-6718.

41. Bi L, Okabe I, Bernard DJ, Wynshaw-Boris A, Nussbaum RL: Proliferative defect and embryonic lethality in mice homozygous for a deletion in the p110alpha subunit of phosphoinositide 3-kinase. The Journal of biological chemistry 1999, 274(16):10963-10968.

42. Network TCGA: Comprehensive molecular portraits of human breast tumours. Nature 2012, 490(7418):61-70. 